\title{
Estructura y composición florística del Bosque Húmedo Tropical de la comunidad de San Jerónimo
}

\section{Structure and floristic composition of the Tropical Rainforest from San Jerónimo Community}

\author{
Adnel Padilla Henry ${ }^{1}$ \\ Loncy Kandler Bendlis ${ }^{2}$ \\ Noé Guadamuz ${ }^{3}$
}

\section{Resumen}

T a biodiversidad forestal es uno de los tópicos más importantes para el manejo forestal sostenible. En Lel 2015, se realizó un inventario forestal aplicando la metodología de Dauber (1995), en la comunidad de San Jerónimo, río Coco, con el propósito de conocer los aspectos florísticos, estructurales, los índices de biodiversidad y proponer alternativas de manejo forestal sostenible.

Se encontró una riqueza florística de 45 familias, 82 géneros y 88 especies. Las familias más representativas fueron Moraceae y Fabaceae. El coeficiente de mezcla fue de (1:3), índice de Shannon (3.84), e índice de Simpson (o.97), lo cual indica una riqueza y heterogeneidad alta.

El 86.87\% de los individuos registrados se agrupó en la clase diamétrica inferior a $39.9 \mathrm{~cm}$ y el $61.71 \%$ de los individuos se encuentra en el estrato medio presentando alturas entre 11 y $20.9 \mathrm{~m}$. Las especies más importante dentro del bosque de acuerdo con el IVI fueron Dialium guianense (aubl) Steud con 35.09 y Tetragastris panamensis Kuntze con 27.84. Se proponen tres alternativas de manejo forestal bajo el modelo de forestería comunitaria: i).-Ordenamiento forestal, ii).-Aprovechamiento forestal y iii).-Modelo de gestión para Pagos por Servicios ambientales.

Palabras clave: biodiversidad forestal; manejo forestal sostenible; inventario forestal; índices de biodiversidad.

\section{Summary}

Forest biodiversity is one of the most important topics for sustainable forest management. In 2015, a forest inventory was carried out applying the methodology of Dauber (1995), in the community of San Jerónimo, Río Coco, with the purpose of knowing the floristic, structural aspects, the biodiversity rating and proposing sustainable forest management alternatives.

A floristic richness of 45 families, 82 genres and 88 species was found. The most representative families were Moraceae and Fabaceae. The mixing coefficient was (1: 3), Shannon index (3.84), and Simpson's index (o.97), which indicates high richness and heterogeneity.

\footnotetext{
1 Ingenierio Agroforestal por la Universidad de las Regiones Autónomas de la Costa Caribe Nicaragüense-Recinto Universitario Bilwi. Correo: padnel@yahoo.es

2 Ingenierio Agroforestal por la Universidad de las Regiones Autónomas de la Costa Caribe Nicaragüense-Recinto Universitario Bilwi. Correo: longecandler@ ymail.com

3 Ingeniero Agroforestal, Profesor de la Universidad de las Regiones Autónomas de la Costa Caribe Nicaragüense-Recinto Universitario Bilwi. Correo: noe.guadamuz@uraccan.edu.ni, 58728408
}

Recibido: 08/02/2017 Aprobado: 23/11/2017 
$86.87 \%$ of the registered individuals were grouped in the diameter class below $39.9 \mathrm{~cm}$ and $61.71 \%$ of the individuals are in the middle stratum, with heights between 11 and $20.9 \mathrm{~m}$. The most important species within the forest according to the IVI were Dialium guianense (aubl) Steud with 35.09 and Tetragastris panamensis Kuntze with 27.84. Three alternatives of forest management are proposed under the community forestry model: i). - Forest management, ii). - Forest exploitation and iii). Management model for payments for environmental services.

Key Words: forest biodiversity; sustainable forest management; forest inventory; biodiversity rating.

\section{Introducción}

La Región Autónoma de la Costa Caribe Norte (RACCN) posee el $43.39 \%$ de los bosques de Nicaragua, y la mayor cobertura se localiza en las comunidades indígenas con el $49 \%$. De acuerdo a los resultados del Inventario Forestal Nacional del 2008 (INAFOR, 2009) son considerados como bosques secundarios debido a los daños causados por el huracán Félix en el 2007 y actualmente por la intervención antropogénica. Si bien es cierto que "los bosques gestionados correctamente poseen un enorme potencial para contribuir al desarrollo sostenible y a una economía más verde" (FAO, 2014), es importante proponer alternativas de manejo mediante un inventario forestal eficiente.

\section{Marco teórico}

\section{Bosque húmedo tropical}

Los bosques húmedos tropicales se encuentran distribuidos en toda América (INIDE, 2014). Estos bosques se clasifican en zonas de vida y numerosos son los bienes y servicios ecosistémicos (Byron $y$ Arnold 1999; Secretariat of the Convention on Biological Diversity 2009; PNUMA 2005). La composición florística está determinada por la riqueza (total de especies observadas en un área) y la diversidad (cantidad de individuos por especie) (UNESCO, PNUMA; FAO, 1980; Sabogal 1994; Hernández 1999; Melo et al, 1997); Otra forma práctica de interpretar la composición florística es el uso de gráficas de curva-especie (Caín et al, citados en Salgado, 1986 Moreno \& Halffter, 2000;
Willott, 2001), a su vez es sencillamente medibles (Van Kuijk et al 2009 citado en (Clavijo, 2012).

\section{Índices de riqueza y diversidad}

Coeficiente de Mezcla (Marmillod, citado por Salgado 1986)

Sirve para medir la intensidad de mezcla de las especies (Becerra 1971; Pielau, 1969 citado en Jhosmar, O, 2009).

Se mide de la siguiente forma:

Donde:

$\mathrm{CM}=$ Coeficiente de Mezcla.

S = Número total de especies en el muestreo

$\mathrm{N}=$ Número total de individuos en el muestreo

$$
\text { C.M. }=\frac{S}{N} \frac{\left(\frac{S}{S}\right)}{\left(\frac{N}{S}\right)}
$$

\section{Índice de Shannon}

Expresa la uniformidad de todas las especies de la muestra. Mide el grado promedio (Magurran, 1988; Peet, 1974; Baev y Penev, 1995) y contempla la cantidad de especies (su riqueza) y la relatividad de los individuos de cada especie (abundancia). (Magurran 2001, citado en Jhosmar, O, 2009). La fórmula está dada por:

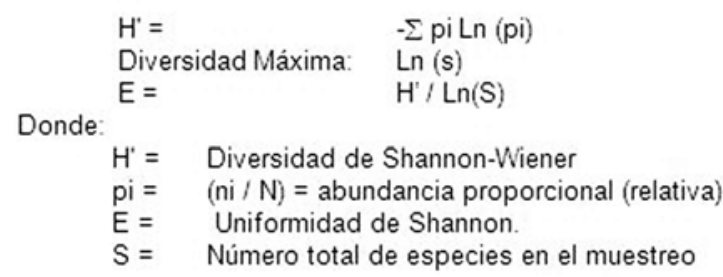

\section{Índice de Simpson}

El índice de Simpson está dado por $\mathrm{D}=\mathrm{p}^{\wedge} 2$ (Pielau, 1969 citado en Jhosmar, O, 2009). Los valores cercanos a uno corresponden a sitios muy diversos (Quesada, 1997, citado en Zamora M, 2010). Se mide de la siguiente manera: 

a. Índice de dominancia de Simpson con la formula $\mathrm{D}=\mathrm{p}^{\wedge} 2$

b. Para el índice de diversidad (1-D).

\section{Estructura del bosque}

Es la organización en el espacio "vertical y horizontal” (Louman, B 2001; Melo y Vargas, 2003); de los individuos que forman un rodal. Los elementos primarios son la forma de crecimiento, la estratificación y la cobertura (Danserau, 1957; Lamprecht, 1990; Valerio et al, 1997). Generalmente la estructura horizontal presenta en forma de "J" invertida, (Monge, 1999; Louman, B 2001); y la estructura vertical, de acuerdo a (Valerio et al, 1997) está determinada por la distribución de los organismos a lo alto de su perfil clasificado en tres estratos (alto, medio y bajo); y constantemente son ocasionados por claros (Monge 1999; Hernández 1999).

\section{Homogeneidad y heterogeneidad}

Mediante la frecuencia se puede calcular el grado de homogeneidad de un bosque, que es un índice fitosociológico creado para conocer la regularidad de la distribución horizontal de cada especie sobre el terreno o su dispersión media en una asociación vegetal (Rosot et al, 1982). Cuando el 80\% o más de los árboles que forma el bosque pertenecen a una misma especie, se trata de un bosque homogéneo, cuando está formado por varias especies forestales es heterogéneo.

\section{Índice de Valor de Importancia (IVI)}

Es la sumatoria de la frecuencia de los porcentajes de abundancia y dominancia, de forma que sea posible comparar el peso ecológico de cada especie dentro de un bosque determinado, y la formula se expresa de la siguiente manera: IVI $=\mathrm{A} \%+\mathrm{F} \%+\mathrm{D} \%$

Donde:

$\mathrm{A} \%=$ abundancia relativa.

$\mathrm{F} \%$ = frecuencia relativa.

$\mathrm{D} \%=$ dominancia relativa.

\section{Manejo forestal}

El Manejo Forestal se define como "la aplicación de métodos administrativos y principios técnicos en una propiedad forestal" (Davis, 1966 citado en Zepeda 1990). Santillán (1986) propone que el manejo forestal es sinónimo de ordenación forestal.

A principios de la década de 1990 surge el concepto de Manejo Forestal Sostenible (MFS) (Poore, 2003), basado en tres pilares fundamentales: económico, social y ecológico o ambiental, (Wiersun 1995, citado en Ros-Tonen et al, 2007).

El Programa Bosques Para Siempre se adhiere a la definición de la Organización Internacional de Maderas Tropicales (OIMT, 1991) definiendo que:

El Manejo Forestal Sostenible es el proceso de administrar en forma permanente la tierra forestal y de lograr uno o más objetivos claramente especificados, para alcanzar un flujo continuo de bienes y servicios deseados del bosque, sin una reducción indebida en sus valores inherentes ni en su productividad futura y sin efectos indebidos no deseables en el ambiente físico y social.

La Ley Forestal vigente No. 462 de Nicaragua, define como el conjunto de acciones y procedimientos que tienen por objeto el cultivo, protección, conservación, restauración o aprovechamiento de los recursos forestales. El Manejo Forestal Sostenible surge bajo el modelo de forestería comunitaria a partir del 2000 en las comunidades de Layasiksa, el bloque SIPBAA, y las Crucetas; promovido por iniciativas de ONG's y comunitarios indígenas.

\section{Alternativas de manejo forestal comunitario}

De acuerdo al Diccionario de la Real Academia Española (2012), la palabra alternativa es sinónimo de "administración, elección, opciones..." En el ámbito del manejo forestal la caracterización y conocimiento de la biodiversidad de los ecosistemas forestales constituye una condición básica para la toma de decisiones sobre el manejo para garantizar una gestión sostenible (Solís, Aguirre, Treviño, Jiménez, Jurado, Corral, 2006). La ley 462, es el encargado de regular la implementación, manejo y aprovechamiento de los recursos forestales en Nicaragua. 


\section{Materiales y método}

\section{Tipo de estudio}

\section{Enfoque cuantitativo y cualitativo.}

\section{Descripción y ubicación del área de estudio}

El área de estudio se localiza en el Municipio de Waspam, Territorio Wangki Li Aubra Tasbaika, RACCN de Nicaragua (Figura 1). En las hojas Cartográficas No: Leimus, 3360 I y Tronquera, 3359 II.

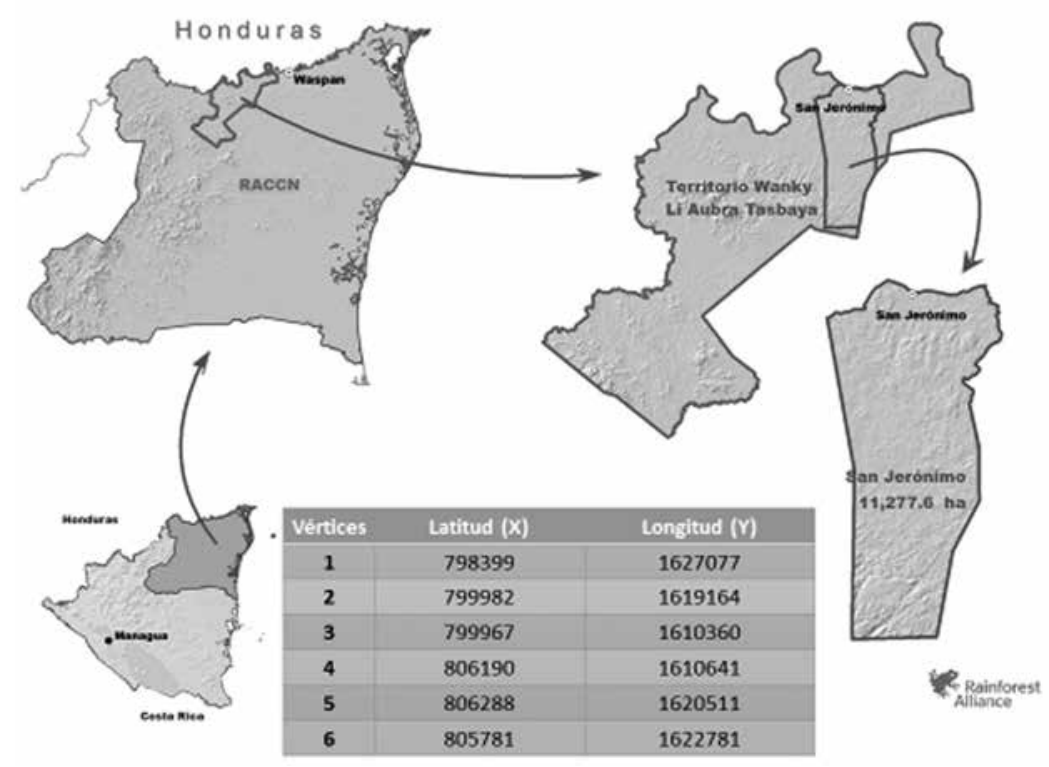

Figura. No. 1 Ubicación geográfica y coordenadas del área de estudio.

Fuente: Elaboración propia (zona 16 Datum WGS84).

\section{Universo y muestra}

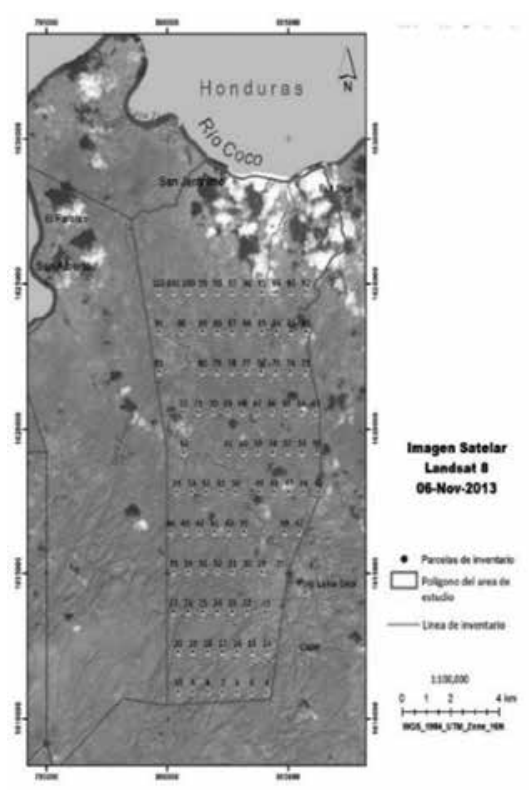

Figura 2. Mapa de distribución y tamaño de las parcelas de inventario. 
El universo fue en un área total de 8500.1 ha y la muestra de 34 ha. Se distribuyó 95 parcelas sistemáticamente. Con intensidad de muestreo de $0.41 \%$ y con un error permisible de $20 \%$ y nivel de confiabilidad al 95\%. Se utilizó mapas a escala de 1:1000oo. Se estableció parcelas rectangulares de $20 \mathrm{~m}$ x 180 m (0.36 ha) distribuidas sistemáticamente, $610 \mathrm{~m}$ entre parcelas y $1380 \mathrm{~m}$ entre fajas de Este-Oeste.

\section{Diseño del inventario}

Se aplicó la Guía práctica y teórica para el diseño de un inventario forestal de reconocimiento (Dauber, 1995) y las (NTON 18 001-04).

\section{Procedimientos metodológicos}

\section{Planificación (pre-campo)}

Se recopiló datos relevantes, a través de entrevistas con los comunitarios, imágenes Landsat 8; cálculos, diseño y georreferenciación de las parcelas de inventario haciendo uso de ARCGIS 10.2 y Excel 2013, siguiendo la metodología de Dauber (1995) y finalmente se diseñó formatos de campo. En la comunidad se realizó reunión y entrenamiento al personal de trabajo. Variables medidas:

- Especie (nombre común miskitu y español).

- Altura total y comercial del árbol en m.

- Diámetro a la altura del pecho (altura de $1.3 \mathrm{~m}$ a partir del suelo)

- Calidad de fuste, grado de iluminación y condición fitosanitario.

- Características topográficas e hidrográficas.

Procesamiento de la información (post-campo)

Una vez digitalizada y completada la información en el programa Microsoft Excel 2013, se procesó la información para analizar e interpretar los resultados. Para la propuesta de las alternativas de manejo se utilizó el análisis florístico y estructural de los inventarios realizados para el presente estudio, de igual manera se compiló información acerca de casos de manejo forestal en Nicaragua.

\section{Parámetros estadísticos y error de muestreo}

Una vez introducido y ordenado la información de campo en el programa Excel, se procesó los datos para obtener el rango aceptable del error de muestreo $\leq$ al $20 \%$, establecido por las normas técnicas. En caso contrario (valor superior al 20\%) volver a replantear las unidades de muestra necesarias a inventariar.

Sin embargo, el procesamiento de los datos determinó un error de muestreo del 9.97\%, como se refleja en el cuadro 1 con un nivel de confiabilidad del $95 \%$. Este resultado es inferior con respecto a los valores que establece las normas técnicas NTON 18-001-04 del INAFOR, la cual estipula que un inventario forestal es confiable y aceptable cuando el error de muestreo es $\leq \mathrm{al} 20 \%$ en función al área basal $(\mathrm{Gm} 2)$.

Cuadro 1. Parámetros estadísticos del error de muestreo.

\begin{tabular}{|l|l|l|l|l|}
\hline \multirow{2}{*}{\multicolumn{1}{c|}{ Parámetros }} & & \multicolumn{3}{c|}{ Especies a partir de 10 cm dap } \\
\cline { 2 - 6 } & & \multicolumn{2}{c|}{ N/ha } & \multicolumn{2}{c|}{ G/ha } & V/ha \\
\hline Tamaño de la parcela & Has & 0.35 & 0.35 & 0.35 \\
\hline Numero de parcelas & UM & 95 & 95 & 95 \\
\hline Intensidad de muestre0 & M\% & 0.41 & 0.41 & 0.41 \\
\hline
\end{tabular}




\begin{tabular}{|c|c|c|c|c|}
\hline \multirow{2}{*}{ Parámetros } & & \multicolumn{3}{|c|}{ Especies a partir de $10 \mathrm{~cm}$ dap } \\
\hline & & N/ha & G/ha & V/ha \\
\hline Media poblacional & $x$ & 2.09 & 0.13 & 0.96 \\
\hline Desviación estándar & Desvest & 1.18 & 0.06 & 0.58 \\
\hline Coeficiente de variación & $\mathrm{CV} \%$ & 56.54 & 48.96 & 60.26 \\
\hline Error estándar & $S$ & 0.12 & 0.01 & 0.06 \\
\hline Varianza de la muestra & S2 & 1.39 & 0.005 & 0.33 \\
\hline \multirow{2}{*}{ Límites de confianza } & Sup.X (+) x (t0.05 x SX) & 2.33 & 0.14 & 1.07 \\
\hline & $\operatorname{Inf} . X(-) \times($ t0.05 x SX) & 1.85 & 0.12 & 0.84 \\
\hline Error de muestreo & $\mathrm{E} \%$ & 11.52 & 9.97 & 12.27 \\
\hline
\end{tabular}

Fuente: Elaboración propia.

\section{Resultados y discusión}

\section{Composición florística}

\section{Riqueza florística}

Se encontraron 88 especies, 82 géneros y 45 Familias. Las familias más representativas fueron la Moraceae con 6 especies (6.82\%), la Fabaceae con 5 especies (5.68\%); la Malvaceae, Leguminosae Papilionoideae, Meliaceae, Leguminosae mimosoideae, Bignoniaceae, todas representadas en 4 especies (4.55\%) cada una; y la familia de la Combretaceae, Clusiaceae, Euphorbiaceae se encuentran distribuidas en 3 especies cada una equivalente a $3.41 \%$.

Las 10 familias con mayor riqueza florística (cuadro 4), representa el $45.45 \%$ con 40 especies. En cambio las 35 familias se encuentran distribuidas en 48 especies representando el 54.55\% de especies en el bosque. De Las 10 familias 6 son representativos en lo observado por (Guadamuz, 2010) en un estudio similar.

Cuadro 2. Riqueza de especie-familia más representativa

\begin{tabular}{|l|l|l|l|}
\hline No. & \multicolumn{1}{|c|}{ Familia } & Especie & \multicolumn{1}{c|}{$\%$} \\
\hline 1 & Moraceae & 6 & 6.82 \\
\hline 2 & Fabaceae & 5 & 5.68 \\
\hline 3 & Malvaceae & 4 & 4.55 \\
\hline 4 & Leguminosae Papilionoideae & 4 & 4.55 \\
\hline 5 & Meliaceae & 4 & 4.55 \\
\hline 6 & Leguminosae Mimosoideae & 4 & 4.55 \\
\hline 7 & Bignoniaceae & 4 & 4.55 \\
\hline 8 & Combretaceae & 3 & 3.41 \\
\hline 9 & Clusiaceae & 3 & 3.41 \\
\hline 10 & Euphorbiaceae & 3 & 3.41 \\
\hline & Total 10 familias más representativos & 40 & 45.45 \\
\hline & 35 FAMILAS & 48 & 54.55 \\
\hline & TOTAL & 88 & 100.00 \\
\hline
\end{tabular}

Fuente: Elaboración propia. 
Estas familias registradas como las más importantes coinciden con estudios realizados por (Defler \& Defler 1996, Cárdenas et al. 1997, Mantilla-Meluk \& Barrios-Rodríguez 1999, Gonzalez 2007), en donde las principales especies de 10 familias, en tres tipos de bosque latifoliado de Costa Rica, fueron: Arecaceae, Combretaceae, Euphorbiaceae, Fabaceae, Lecythidaceae, Moraceae, Myristicaceae, Sapotaceae, Urticaceae y Vochysiaceae. Otro planteamiento por Gentry $(1988,1992)$, concuerda que los bosques húmedos tropicales a nivel mundial son consistentemente dominados por un pequeño grupo de familias encabezados por las leguminosas y las Fabaceae.

Ter Steege et al (200o), también confirmaron el planteamiento de Gentry (s/f), al hallar que el $16 \%$ de los individuos pertenecen a dicha familia, caracterizando de esta manera que los bosques húmedos tropicales presentan ambientes propicios para las familias más importantes encontradas en el presente estudio. Este resultado de la riqueza familiar y de especies, en concordancia con Sabogal (1994), ha determinado la riqueza florística del bosque en el presente estudio.

\section{Curvas de especies}

La riqueza florística evaluada mediante la curva de especies, indicó un fuerte incremento hasta la parcela 33 (figura 3), hasta lograr definirse la línea asintótica desde la parcela 83 finalizando en las 95 parcelas. Concluyendo que aunque se aumente el esfuerzo de muestreo no será posible encontrar nuevas especies (Soberon y Llorente, 1993; Moreno y Halffter, 2000; Gotelli \& Colwell, 2001).

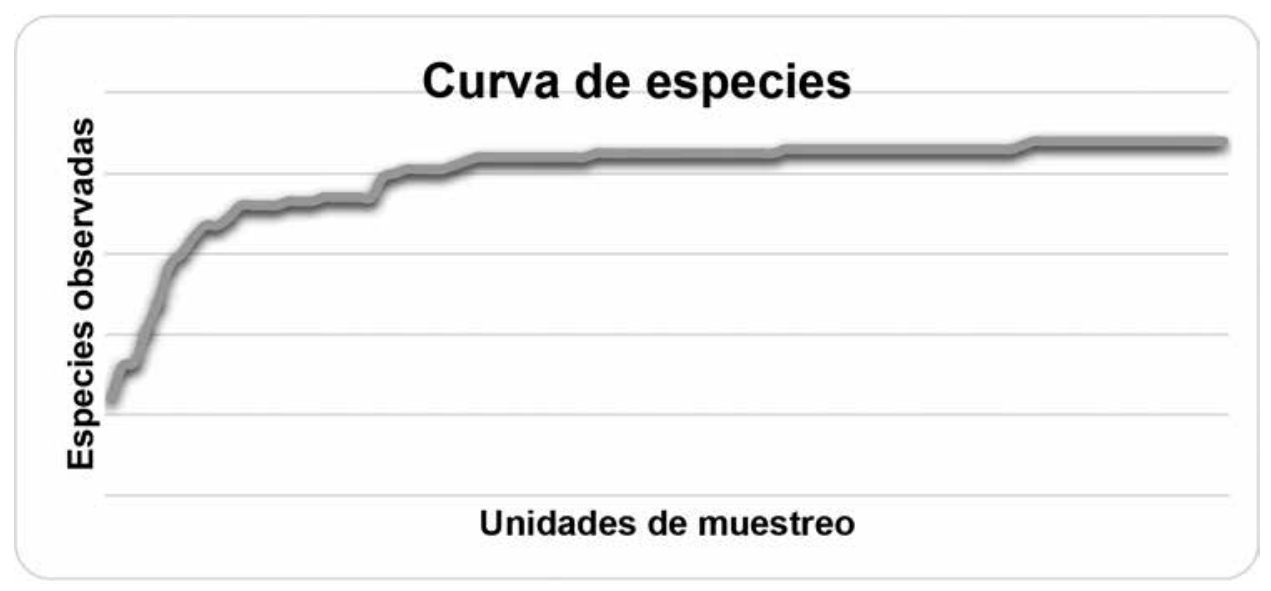

\section{Diversidad florística}

\begin{tabular}{|l|l|l|}
\hline \multicolumn{1}{|c|}{ Índices de biodiversidad } & Valor & \multicolumn{1}{c|}{ Caracterización } \\
\hline Coeficiente de mezcla (CM) & $1: 3$ & $\begin{array}{l}\text { Alto grado de mezcla, 1 especie cada 3 individuos. } \\
\text { Varía en bosques amazónicos (1:3 y 1:4) Lamprecht (1990), Colombia, similar a la zona de es- } \\
\text { tudio, registró valores de 1:3,98 (Melo y Vargas, 2003). }\end{array}$ \\
\hline Índice de Shannon-Wiener (H) & 3,84 & $\begin{array}{l}\text { Alta diversidad; Magurran (1988) este índice varía entre 1.5 y 3.5, rara vez 4.5; valores cercanos } \\
\text { a 3,5 reflejan sitios muy diversos. } \\
\text { Importancia biológica, ecológica y genética; para el bienestar humano y faunística. }\end{array}$ \\
\hline Índice de Simpson (D) & 0.03 & $\begin{array}{l}\text { Baja dominancia, por lo tanto diversidad alta (0.97), presenta ecosistema funcional (Guada- } \\
\text { muz, 2010). Aunque desaparezca la función ecológica de una especie en una población puede } \\
\text { ser compensada por otras especies (Louman B, 2001). }\end{array}$ \\
\hline
\end{tabular}


La diversidad florística obtenida mediante el coeficiente de mezcla, para el conjunto de árboles con $\mathrm{DAP} \geq 10 \mathrm{~cm}$, fue de 1:3, lo que indica que el bosque estudiado presenta un alto grado de mezcla con la aparición, en promedio, de una nueva especie cada 3 individuos. Lamprecht (1990) señaló que para bosques amazónicos el coeficiente de mezcla varía entre 1:3 y 1:4, el cual tiene mucha similitud con el bosque estudiado. Otros estudios realizados en un bosque húmedo de colinas bajas ubicado en la región del litoral Pacífico de Colombia, se asemeja a la zona del presente estudio, en donde se registró un valor de 1:3,98 (Melo y Vargas, 2003).

De acuerdo a Magurran (1988) el índice de diversidad de Shannon-Wiener varía entre 1,5 y 3,5; y rara vez alcanza valores de 4,5, por lo que valores cercanos a 3,5 reflejan sitios muy diversos. La alta diversidad del índice $\mathrm{H}$ está referida a las especies que interactúan en su ambiente indicando principalmente la diversidad biológica que posee. En este sentido la diversidad biológica forestal que posee el bosque de la comunidad aporta en el bienestar humano mediante la disponibilidad de una multitud de servicios de los ecosistemas, tales como la purificación del agua, el suministro de oxígeno, y los beneficios espirituales y culturales. Otra caracterización de la alta diversidad forestal que sugiere el índice $\mathrm{H}$ es el potencial hábitat que ofrece para las especies faunísticas.

El índice de Simpson arrojó un valor de 0.03 (dominancia baja). Este resultado es similar a un caso observado en el bosque húmedo tropical ubicado en la Zona de la Cangreja, Colombia, (Acosta, 1998), por lo que el sitio se caracteriza como altamente diverso con 0.97. De acuerdo a (Louman B, 2001), varias especies vegetales pueden tener la misma función ecológica; sin embargo, el aprovechamiento permite la reducción en la población de una especie y que puede ser compensada por un incremento en la población de otras especies con la misma función. Desde esta perspectiva el bosque puede ser destinado al aprovechamiento responsable.

\section{Descripción de la estructura del bosque}

\section{Estructura Horizontal}

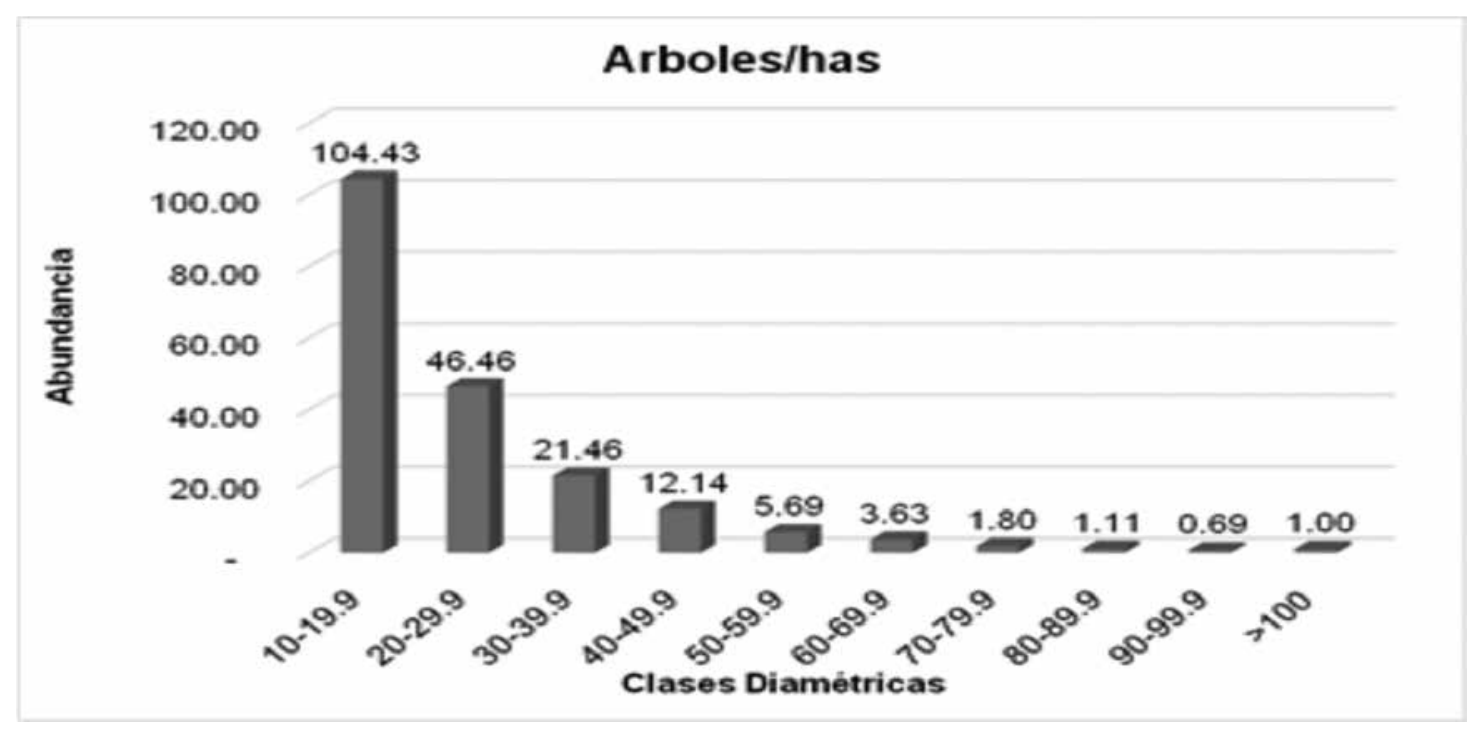

Fuente: Elaboración propia

Figura 5. Abundancia (arb/ha) de las clases altimétricas

El bosque presenta una "J invertida" (Figura 4) con mayor presencia de individuos en las clases diamétricas $\leq 39.9 \mathrm{~cm}$ con un $86.87 \%$. Posiblemente por la presencia de muchos claros...Palacios y Ramos (1999). 


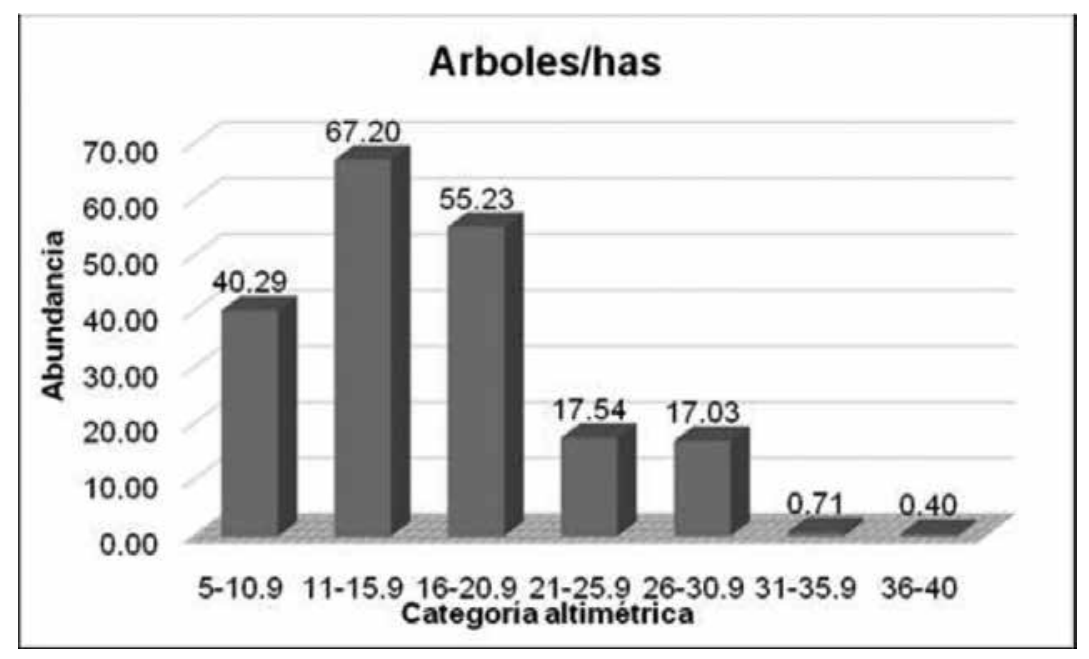

El área de estudio posee las características descritas por Palacios y Ramos debido a los individuos que se están regenerándose rápidamente.

\section{Estructura vertical}

Los diferentes pisos (figura 5) demuestra que hay mayor presencia de individuos entre 11 y $20.9 \mathrm{~m}$. con un $61.71 \%$. Probablemente porque han aprovechado las perturbaciones (Monge, 1999). La gráfica muestra una caracterización adecuada del bosque con respecto a su perfil vertical Hernández (1999).

Valerio et al (1997), menciona que el bosque está dividido en tres estratos; por tanto la distribución vertical se distribuyó en: dosel bajo (5-10.9 $\mathrm{m}$ con 20.31\%), el dosel medio (11-20.9 $\mathrm{m}$ con 61.71\%) y el dosel alto (21-40 $\mathrm{m}$ con 17.99\%).

\section{Índice de Valor de Importancia}

De acuerdo a (Lamprecht, 1990, citado por Salgado 1996), mediante el “Índice de Valor de Importancia” (IVI), permite obtener información acerca de las especies que mejor se establecen en la zona.

Cuadro 5. Especies con mayor Índice de Valor de Importancia

\begin{tabular}{|l|l|l|l|l|l|l|c|}
\hline No. & \multicolumn{1}{|c|}{ Nombre científico } & Nombre común & Nombre miskitu & Abun $\%$ & Dom \% & Frec \% & IVI 100\% \\
\hline 1 & Dialium guianense (aubl) Steud & Comenegro & Slimh & 7.07 & 6.58 & 91.58 & 35.08 \\
\hline 2 & Tetragastris panamensis Kuntze & Kerosen & Sahkal & 3.49 & 2.14 & 77.89 & 27.84 \\
\hline 3 & Luehea seemannii & Guácimo colorado & Kira & 2.69 & 6.13 & 70.53 & 26.45 \\
\hline 4 & Cecropia insignis liemb & Guarumo & Planh & 6.86 & 2.48 & 65.26 & 24.87 \\
\hline 5 & Gliricidia Sepium & Madero negro & Tasmuk & 4.55 & 3.05 & 63.16 & 23.59 \\
\hline 6 & Ochroma lagopus & Balsa/guano & Puhlak & 7.63 & 3.96 & 55.79 & 22.46 \\
\hline 7 & Spondia purpuria & Jovo & Pahra & 2.28 & 2.73 & 62.11 & 22.37 \\
\hline 8 & Hyeronima alchorneoides Allen. & Nancitón & Kiaki dusa & 1.56 & 4.27 & 58.95 & 21.59 \\
\hline 9 & Grias cauliflora L & Bul & 2.90 & 1.73 & 58.95 & 21.19 \\
\hline 10 & Terminalia oblongán & Labina & 2.71 & 3.58 & 56.84 & 21.04 \\
\hline & Subtotal de las 10 especies más importantes & & 41.73 & 36.66 & 27.99 & 28.86 \\
\hline & Resto de las 78 Especies & & 58.27 & 63.34 & 72.01 & 71.14 \\
\hline & TOTAL & & 100.00 & 100.00 & 100.00 & 100.00 \\
\hline
\end{tabular}

Fuente: Elaboración propia 
En el cuadro 5 se observa que la especie Dialium guianense (aubl) Steud presenta una distribución amplia y uniforme en toda el área, siendo abundante con el $7.07 \%$, dominancia con $6.58 \%$ y frecuencia con un $91.58 \%$. Es decir, que al recorrer el bosque la especie con más probabilidad de ser observada es esta especie, en este sentido posee un IVI de 35.09, lo que hace prever que es una especie con alta capacidad de adaptarse a diferentes condiciones (lumínicas, suelo, pendiente, otras).

Otro comportamiento similar es la especie Tetragastris panamensis Kuntze que presenta un IVI del $27.84 \%$. Por otro lado Luehea seemannii es otra de las especies que ocupa el tercer lugar reportando un IVI los porcentajes de $26.45 \%$, de abundancia baja de 2.69 , pero dominancia alta con 6.13 y frecuencia de 70.53 , esto demuestra que tiene gran capacidad para aprovechar los nutrimentos disponibles y desarrollarse a plenitud.

Esto no quiere decir que es la especie que mejor se está desarrollando dentro del bosque, porque existen otras especies que no necesitan tener grandes dimensiones para satisfacer sus necesidades (esciófitas totales); pero si se puede asegurar que es una de las especies que más está creciendo dentro del bosque además de las otras siete especies importantes en el IVI.

\section{Alternativas para el manejo forestal sostenible}

Las alternativas de manejo forestal buscarán implementar el uso responsable de los bienes y servicios disponibles. Para esto se buscará alianzas con organismos internacionales y empresas privadas que promueven el uso sostenible de los recursos naturales. Desde esta perspectiva se espera las iniciativas de autogestión y mayor interés de parte del gobierno territorial y la comunidad.

\section{Ordenamiento forestal}

Las diversas demandas se pueden satisfacer mediante una separación espacial de áreas con diferentes usos (Vincent et al, 1993; Binkley 1997; Zhang 2005) o mediante el manejo integrado. El ordenamiento forestal consiste en categorizar en diferentes usos de suelo. Esta propuesta está enfocada principalmente en la producción local utilizando las áreas de barbecho (cuadro 6).

Cuadro 6. Áreas de uso e implementación de acciones para la alternativa de ordenamiento forestal

\begin{tabular}{|c|c|c|c|}
\hline Categorización & Superficie (has) & Acciones & Objetivos \\
\hline $\begin{array}{l}\text { Bosque de cobertura } \\
\text { forestal }\end{array}$ & 8500.1 & $\begin{array}{l}\text { Planificación para el monitoreo a nivel comunal en coordina- } \\
\text { ción con gobiernos municipales y territoriales. } \\
\text { Gestión de proyectos ambientales y agroforestales especial- } \\
\text { mente sistemas taungya }\end{array}$ & - Protección y conservación. \\
\hline $\begin{array}{l}\text { Barbecho: } \\
\text { Agricultura } \\
\text { Ganadería }\end{array}$ & $2,075.2$ & $\begin{array}{l}\text { Plan de capacitación para la implementación de prácticas agro- } \\
\text { forestales ( Sistemas agrosilvopastoriles, sistemas Silvopasto- } \\
\text { riles, entre otras. }\end{array}$ & $\begin{array}{l}\text { - Mejorar la producción ganadera } \\
\text { - Tecnificar los sistemas productivos en culti- } \\
\text { vos anuales, frutales y temporales. } \\
\text { - Disminución de prácticas convencionales de } \\
\text { la rosa, tala y quema. }\end{array}$ \\
\hline Bosque Ripario & 529.3 & Proyectos de reforestación y PSAH & Proteger fuentes de agua. \\
\hline Zona poblada & 173 & Siembra de cercas vivas entre comunidad y bosque. & Reorganizar y mantener el área poblada. \\
\hline
\end{tabular}

Fuente: elaboración propia

\section{Aprovechamiento forestal}

Esta alternativa está dirigida al aprovechamiento de las especies forestales bajo el sistema policíclico el cual debe estar planificado de la siguiente manera: 
Cuadro 7. Resultados preliminares para el aprovechamiento forestal

\begin{tabular}{|l|l|}
\hline \multicolumn{1}{|c|}{ Descripción } & \multicolumn{1}{c|}{ Valores } \\
\hline Superficie de bosque productivo & 8500.1 \\
\hline Ciclo de Corta & 20 años \\
\hline Área de Corta Anual & Promedio 425 ha \\
\hline Producción anual Productos forestales & \\
\hline Especies maderables (ACom) & $2902.4 \mathrm{~m} 3$ \\
\hline Especies maderables (PCom) & $1033.8 \mathrm{~m} 3$ \\
\hline Especies no maderables (NCom) (Bambú, tuno y Guano) & $600.5 \mathrm{~m} 3$ \\
\hline Especies no maderables (NCom) (Caucho) & $73 \mathrm{lnd} / \mathrm{ha}$ \\
\hline
\end{tabular}

Fuente: Resultados del Inventario realizado en el presente estudio.

La propuesta para el mercado local de las especies maderables de poca demanda debe ser promovida con mayor interés comercial; y los productos maderables no comerciales, como el guano, tuno, caucho y bambú, deben estar incluidos en el mercado para el aprovechamiento local y municipal.

Cuadro 8. Plan de acción y objetivos del aprovechamiento forestal

\begin{tabular}{|l|l|l|l|}
\hline Categoría & $\begin{array}{c}\text { Superficie } \\
\text { (ha) }\end{array}$ & \multicolumn{1}{|c|}{$\begin{array}{c}\text { Gestiones a } \\
\text { realizar }\end{array}$} & \multicolumn{1}{|c|}{ Objetivos } \\
\hline Bosque & 8500.1 & Gestión del PGMF. & Gestión del POA. \\
Gestión del ElA. & $\begin{array}{l}\text { Aprovechar Responsable y } \\
\text { eficientemente los productos } \\
\text { maderables. }\end{array}$ \\
\hline
\end{tabular}

Fuente: elaboración propia.

Pago por Servicios Ambientales (PSA/PSAH)

Esta alternativa consiste en las diferentes actividades mediante el cual buscará mantener la integridad del ecosistema forestal.

- Promover proyectos piloto REDD.

- Elaborar y gestionar proyectos de Pago por Servicios Ambientales Hídricos.

Propuesta de acciones a ejecutarse en el marco de las alternativas de manejo del recurso forestal.

\section{Cuadro 9. Acciones para la implementación del manejo forestal comunitario}

\begin{tabular}{|l|l|}
\hline \multicolumn{1}{|c|}{ Líneas de acción } & \multicolumn{1}{|c|}{ Responsables/ Participantes } \\
\hline $\begin{array}{l}\text { 1-. Plan de Capacitación en temas de: } \\
\text { a-. Gobernanza indígena o territorial. }\end{array}$ & GTI, Gobiernos comunales, miembros de la \\
b-. Manejo foresta. & comunidad (Hombres y mujeres). \\
c-. Monitoreo de flora y fauna. & \\
d-. Otros más en relación a los temas de interés. ambiental, social, económico. & \\
\hline $\begin{array}{l}\text { 2-. Reestructuración } \\
\text { a-. Reorganización Territorial/comunal con el fin de formar comisiones de Monitoreo y protección de los } \\
\text { recursos naturales. } \\
\text { b-. Crear normativas internos de la comunidad en función a las alternativas de manejo forestal. }\end{array}$ & $\begin{array}{l}\text { GTI, Gobiernos comunales, Regente forestal } \\
\text { y miembros de la comunidad. }\end{array}$ \\
\hline
\end{tabular}

Fuente: elaboración propia. 


\section{Conclusiones}

- El bosque presenta una estructura florística muy representativa debido a que existe una alta riqueza de 88 especies, 82 géneros y 45 familias; similar a bosques no intervenidos y con una heterogeneidad significativa (Coeficiente de Mezcla de 1:3, Índice de Shannon-Weaner $\mathrm{H}=3.84$ e Índice de Simpson $\mathrm{D}=0.97$ ).

- La riqueza y diversidad del bosque es potencialmente importantes para el refugio de vida silvestre, conservación de especies en peligro de extinción (flora y fauna), protección de fuentes hídricas, suelos y cerros; además de ser atractivo para el manejo forestal.

- Es un bosque altamente productivo ecológica y económicamente ya que presenta una estructura horizontal de $\mathrm{J}$ invertida, donde el $86.87 \%$ se agrupan en las clases diamétricas $\leq$ a $39.9 \mathrm{~cm}$, y la estructura vertical presenta una abundancia del $61.71 \%$ de los individuos en el rango de 11 a $20.9 \mathrm{~m}$ de altura.

- El bosque presenta óptimas condiciones para las especies Heliófitas y Esciofitas, indicando un desarrollo equilibrado, los más representativos son: comenegro, guácimo colorado, kerosen, Guarumo, madero negro, guano, jocote jovo, nancitón, tabacón y guayabón.

- Las alternativas de uso potencial que presenta el bosque están dirigidas al aprovechamiento sostenible del recurso maderable y a la inserción de procesos de pago por servicios ambientales.

- Las propuestas de las alternativas de manejo son viables tanto ecológica y económicamente para el área de bosque estudiado.

\section{Recomendaciones}

- Establecer medidas de protección para evitar la migración de terceros, incendios forestales y otras actividades que afectan negativamente el recurso bosque.
- Monitorear los cambios en la composición florística y estructura del bosque.

- Gestionar recursos técnicos y financieros.

- Gestionar acuerdos y convenios con ONG's, instituciones y empresas para garantizar la sostenibilidad.

- Crear convenios con las universidades URACCAN y BICU-CIUM, para dar seguimiento este tipo de estudio.

\section{Lista de referencias}

Cesar Sabogal et al (2001). Aprovechamiento Forestal Mejorado en Bosques de Producción: Estudio De Casos Los Filos, río San Juan, Nicaragua. / Turrialba, C. R: CATIE. Unidad De Manejo De Bosques Naturales. 57 P.; 27 Cm.(Serie Técnica. Informe Técnico/ Catie; No. 323.

Cordero, W. (1989). Aprovechamiento Forestal. Cartago, Costa Rica Institución Tecnológico De Costa Rica. Serie De Apoyo Académico No. 8.26 P.

Clavijo, G. A. (2012). Identificación y caracterización de tipos de bosques en la Zona de Usos. Turialba, Costa Rica.

Dauber, Herhard. 1995. Guía práctica y teórica para el diseño de un inventario forestal de reconocimiento. Santa Cruz, Bolivia.

FAO. (2014). El estado de los bosques del mundo. 1-2.

Frank H. Wadsworth (1997). Producción Forestal Para América Tropical. U.S. Dept. Of Agriculture, Forest Service. Washington, Dc $563 \mathrm{P}$

García-Montiel DC. (2002). El legado de la actividad humana en los bosques neotropicales contemporáneos. En: Guariguata MR, Kattan GH (eds) Ecología y Conservación de Bosques Neotropicales Libro Universitario Regional (EULAC-GTZ). Cartago, Costa Rica. 
Guadamuz, N. (2010). Regeneracion natural del bosque huracanado de la finca Snaki, comunidad de Moss pam a tres años del Huracán Félix. Waspam, Region Autonoma de la Costa Caribe de Nicaragua, Nicaragua.

Johnson Hugh. El Bosque (Fauna, Flora Y Recursos Económicos del Bosque Mundial). 1981. Milanesa, 21-23. 08017, Barcelona. Editorial Blume.

Lamprecht, Hans (1990). Silvicultura en los trópicos: los ecosistemas forestales en los bosques tropicales y sus especies arbóreas; posibilidades y métodos para un aprovechamiento sostenido. Trad. Del Antonio Carrillo. Deutsche Gesellschaft Für Technische Zusammenarbeit (Gtz) Gmbh.- Rossdorf: Tz - Verl. - Ges.

Louman, B.; Quirós, D.; Nilsson, M. (2001). Silvicultura de bosques latifoliados húmedos con énfasis en América Central. Turrialba, Costa Rica, CATIE. Serie técnica, Manual técnico No 46. $256 \mathrm{p}$.

LOZANO, G. (1996). Notas sobre biodiversidad. Instituto de ciencias naturales. Bogotá, Colombia.

MALLEUX J. (1982). Manual del Técnico Forestal. Cochabamba Bolivia. 37-39 pp.

Navarro, C. y Navarro, J. 1999. Estudio de la composición florística mayor de $10 \mathrm{~cm}$ de DAP en bosque húmedo tropical, Laboratorio Natural. Tesis Ingeniería Forestal. Honduras. Centro Universitario Regional del Litoral Atlántico.

NICARIBE. (2012). Política de actuación en relación con pueblos indígenas-FIDA. RACCN, Puerto Cabezas.
Palacios, W. (2004). Los Gremios forestales en los bosques tropicales húmedos del Ecuador. (En línea). Consultado agosto 30 de 2007. Disponible en http://www.lyonia.org.

Quiróz, D; Gomez, M. (1997). Manejo sustentable de un bosque natural Trópico en Costa Rica. Análisis Financiero. Turrialba, Costa Rica, CATIE. Serie Técnica. Informe Técnico No. 225. Colección De Silvicultura Y Manejo De Bosques. p. 22.

Reyes Rodas, R. (1995). Caracterización y evaluación de la sostenibilidad de los sistemas de producción de la Concesión Comunitaria De San Miguel, Petén, Guatemala. Tesis Mag. Sc. Catie, Turrialba, C. R. p. 186.

Torres, J. (2002). Estudio florístico estructural de una asociación vegetal en el bosque latifoliado maduro de la Montaña de El Uyuca. Tesis Ingeniería Agronómica. Honduras. Escuela Agrícola Panamericana "El Zamorano".

Venegas, J. (2000). Caracterización de la estructura vertical y horizontal en bosques de pino-encino. Nuevo León, México. p. 105.

White, P. S.,\& S. T. A. Pickett. (1985). Natural Disturbance And Patch Dynamics: An Introduction. Pp. 3-13 En S. T. A. Pickett \& P. $S$. White, Editores. The Ecology Of Natural Disturbance And Patch Dynamics. Academic Press, Usa.

World Resources Institute (2006). Instituto de Recursos Mundiales. Ecosistemas y Gente. (En línea). .Consultado julio 20 de 2007. Disponible en http://www.wri.org.

Zamora, M (2010). Caracterización de la flora y estructura de un bosque transicional húmedo a seco. Miramar, Puntarenas, Costa Rica. p. 116. 